\title{
Es braucht mehr Fairness
}

Thomas Mattig

Direktor Gesundheitsförderung Schweiz

Das komplette Interview das Thomas Mattig mit Sir Michael Marmot, Professor für Epidemiologie und öffentliche Gesundheit am University College London, geführt hat, finden Sie auf www.gesundheitsfoerderung.ch

Korrespondenz: Thomas Mattig Gesundheitsförderung Schweiz Dufourstrasse 30 CH-3000 Bern 6 Tel. 0313500404 Fax 0313501700
Wie gross ist der Einfluss, den der soziale Status und das Lebensumfeld eines Menschen auf seine Gesundheit haben? Mit dieser Frage beschäftigte sich die Kommission für soziale Gesundheitsdeterminanten der Weltgesundheitsorganisation (WHO). Und kommt zu klaren Schlüssen: Der Einfluss ist gross. Um die Problematik anzugehen, brauche es «mehr Fairness und ein ganzheitliches politisches Vorgehen». Chancengleichheit wird auch ein Thema der Weltkonferenz der Gesundheitsförderung im Juli 2010 in Genf sein.

Das Thema der sozialen Determinanten der Gesundheit bewegt und ist hochaktuell. Zu Beginn dieses Jahres haben wir an unserer 11. Nationalen Gesundheitsförderungs-Konferenz in Pfäffikon intensiv darüber diskutiert. Als Organisatorin der 20. Weltkonferenz für Gesundheitsförderung vom 11. bis 15. Juli 2010 in Genf (siehe Kasten) werden wir daran anknüpfen mit der Frage, wie Gesundheit, Chancengleichheit und nachhaltige Entwicklung zusammenhängen.

20. Weltkonferenz der Gesundheitsförderung in Genf: Gesundheit, Chancengleichheit und nachhaltige Entwicklung

Vom 11.-15. Juli 2010 findet in der Schweiz unter dem Titel «Gesundheit, Chancengleichheit und nachhaltige Entwicklung» erstmals eine Weltkonferenz zum Thema Gesundheitsförderung statt. Die Internationale Union der Gesundheitsförderungsorganisationen sowie die Gesundheitsförderung Schweiz als Gastgeber erwarten über zweitausend Fachleute und Entscheidungsträger(innen) aus der ganzen Welt, die in den Bereichen Gesundheitsförderung, öffentliches Gesundheitswesen und nachhaltige Entwicklung tätig sind.

An der Konferenz sollen unter anderen folgende Fragen diskutiert werden: Wie wirken sich Mobilität und Verstädterung auf die Gesundheit aus? Wie prägen sie unseren Lebensstil? Wie beeinflussen Migration und Wertkonflikte unsere Welt und Gesundheit? Welcher Stellenwert kommt dem Klimawandel und der aktuellen Weltwirtschaftskrise zu?

Zur Förderung der Zusammenarbeit, des Dialogs und des Austauschs sind vor, während und nach der Konferenz diverse Nebenveranstaltungen geplant. Unter dem Motto «Take a Walk on the Wild Side» kommen kreative und interaktive Lernmethoden zum Zuge, beispielsweise «Open Space» und moderierte Präsentationen von elektronischen

\section{Arbeit, Bildung und gute Gesundheit}

Für den Vorsitzenden der WHO-Kommission für soziale Determinanten der Gesundheit, Professor Sir Michael Marmot, ist klar, dass Fairness im Kontext der Gesundheitsförderung «das Schlüsselelement» ist. Für die Bevölkerung und jeden Einzelnen sei es wichtig, eine Gesellschaft anzustreben, «in der jeder eine gleiche Chance auf gute Gesundheit, gute Bildung und eine anständige Arbeit hat». Im persönlichen Gespräch mit ihm weist der Professor für Epidemiologie und öffentliche Gesundheit am University College London auf die Vielschichtigkeit der Problematik hin. Die beginnt nämlich schon bei der Definition von Gesundheit. Jene der WHO hebe die Tatsache hervor, dass Gesundheit nicht nur die Abwesenheit von Krankheit beinhalte, sondern auch eine positive Vorstellung, nämlich die Freiheit, «zu tun und zu sein». Konkret: Gesundheit hängt davon ab, wieviel Einfluss- und Gestaltungsmöglichkei-

Postern, um den Teilnehmenden einzigartige, anregende Lernerfahrungen zu bieten und ihnen zu helfen, sich für andere Fachgebiete und Netzwerke zu öffnen. Ergänzend dazu ermöglichen wissenschaftliche und gesellschaftliche Anlässe den Teilnehmenden ein optimales Networking. Abstracts (für Symposien, Workshops, mündliche Präsentationen und innovative Session-Formate) können seit dem 31. August 2009 eingesandt werden. Die Abstracts sollen Kernfragen der Gesundheitsförderung und die entscheidenden Wirkungszusammenhänge zwischen Gesundheit und Umweltveränderungen behandeln. Die Vergabe der verfügbaren Stipendien ist inhaltlich und zeitlich eng an das Auswahlverfahren für Abstracts geknüpft, das bis Februar 2010 abgewickelt wird.

Die Konferenz findet im Centre International de Conférences Genève statt. Für Teilnehmende wurden in Hotels verschiedener Kategorien grosse Zimmerkontingente zu vergünstigten Preisen reserviert. Von allen Hotels aus gelangt man mit dem öffentlichen Verkehr leicht ins Kongresszentrum (Benützung für Konferenzteilnehmende kostenlos).

Regelmässig aktualisierte Informationen zu Themen, Referenten und Veranstaltungen sind auf der offiziellen Website www.iuhpeconference.net verfügbar, auf der sich Interessenten auch für die Zusendung des elektronischen Konferenz-Newsletter anmelden können. 
ten der Mensch auf seine Arbeits- und Lebensumstände hat.

Diese Einflussmöglichkeiten sind in armen Ländern offensichtlich viel geringer als in reichen Ländern: Wer kein sauberes, fliessendes Wasser hat und 8 Kilometer zu Fuss gehen muss, um es zu holen, der vermag sein Leben nur bedingt zu gestalten. Gleiches gilt für Menschen, die zuwenig Geld haben, um ihre Kinder zu ernähren. Das lässt aber nicht nur den einfachen Schluss zu, dass es den Reichen besser geht als den Armen. Logisch sei auch, dass jene auf der zweituntersten Stufe eine bessere Gesundheit haben als jene auf der untersten Stufe. «Und jene auf der zweiten Stufe sind gesünder als die auf der dritten Stufe.»

\section{Die Problematik geht uns alle an}

Genau deshalb, sagt Marmot, gehe die Problematik der sozialen Determinanten der Gesundheit nicht nur Menschen in Entwicklungsländern etwas an, sondern auch jene in den Industrienationen. Menschen der untersten sozialen Schichten in der Schweiz oder Grossbritannien sterben nicht an Cholera, Malaria oder Diarrhoe. Sie sterben an Herzkrankheiten, Krebs und an Demenz. Also an denselben Dingen wie Leute der obersten Schichten. Aber sie sterben früher. Marmots Studien in der öffentlichen Verwaltung Grossbritanniens haben ergeben, dass Leute, die als höherqualifizierte Berufsleute arbeiten, aber nicht oberste Führungsposition bekleiden, gesundheitlich angeschlagener sind und eine höhere Sterberate aufweisen als ihre Vorgesetzten.

In den abschliessenden Empfehlungen fordert die WHO-Kommission Verbesserungen in Bezug auf die Bedingungen des täglichen Lebens und betont die Wichtigkeit der Ausbildung und Forschung. Dabei geht es vor allem um Fortschritte im Bereich der Bildung, Beschäftigung, frühkindlichen Erziehung oder der städtischen Verhältnisse. Dazu brauche es nicht nur Anstrengungen aller Organisationen und Politiker aus dem Gesundheitsbereich, sondern ein «ganzheitliches politisches Vorgehen».

\section{Die 20. Weltkonferenz}

\section{der Gesundheitsförderung in Genf}

An der 20. Weltkonferenz der Gesundheitsförderung, die vom 11. bis 15. Juli 2010 zum ersten Mal in der Schweiz stattfindet und von Gesundheitsförderung Schweiz im Auftrag des Weltverbands der Gesundheitsförderung (IUHPE) organisiert wird, stellen wir die Themen Gesundheit und Chancengleichheit bewusst in Bezug zu den Anliegen einer nachhaltigen Entwicklung. Wie können gesündere und nachhaltigere Lebensweisen - im Zeichen der voranschreitenden Globalisierung und Urbanisierung - effizient und wirkungsvoll gefördert werden? Wie können wir den aufkommenden Solidaritätsverlust und soziale Ungerechtigkeiten innerhalb und zwischen den Ländern aufhalten? Die Konferenz wird - so hoffen wir - erste Antworten skizzieren und die internationale Diskussion weiter voranbringen. Wir rechnen mit zahlreichen Teilnehmenden aus der ganzen Welt, auch über den Gesundheitssektor hinaus. Denn eine nachhaltige Verbesserung von Gesundheit und Wohlbefinden in einer globalisierten und unter Ressourcenverknappung leidenden Welt braucht starke, übergreifende Partnerschaften.

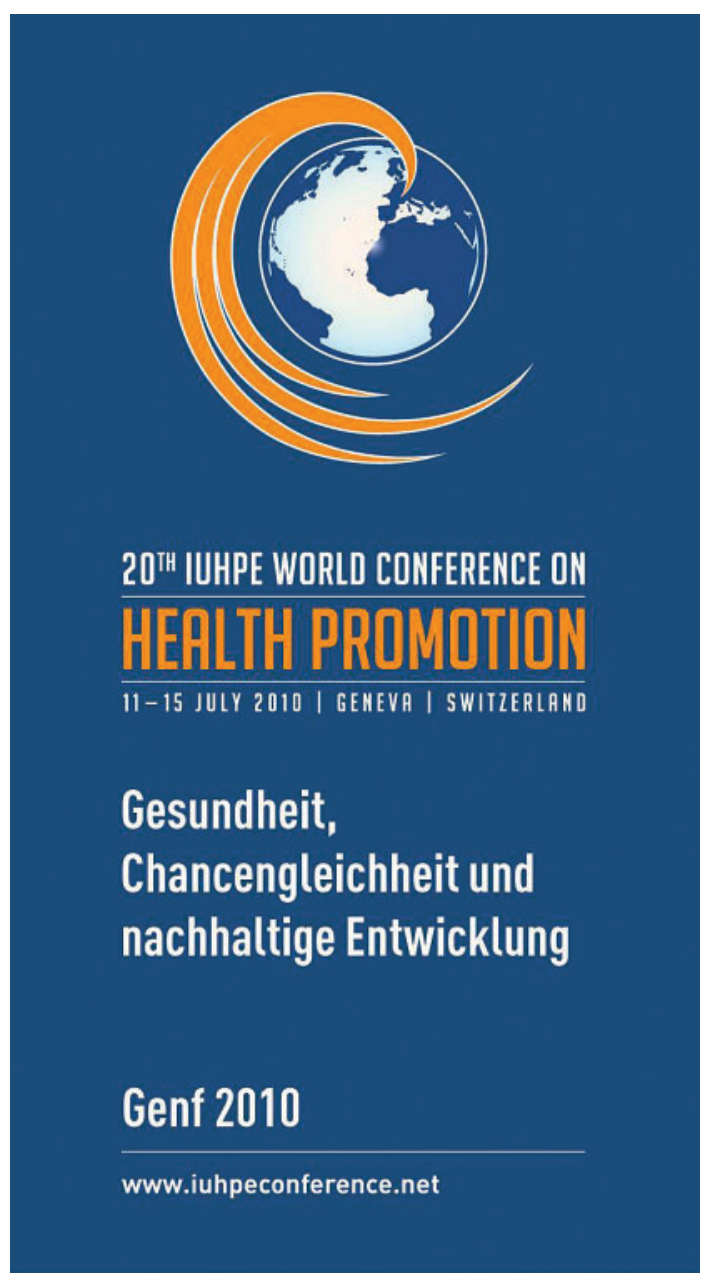

